
2 Research Square
Preprints are preliminary reports that have not undergone peer review.
They should not be considered conclusive, used to inform clinical practice, or referenced by the media as validated information.

\title{
miRNA-485-5p, an Inflammation-Related microRNA, May be a Diagnostic Biomarker of Rheumatoid Arthritis
}

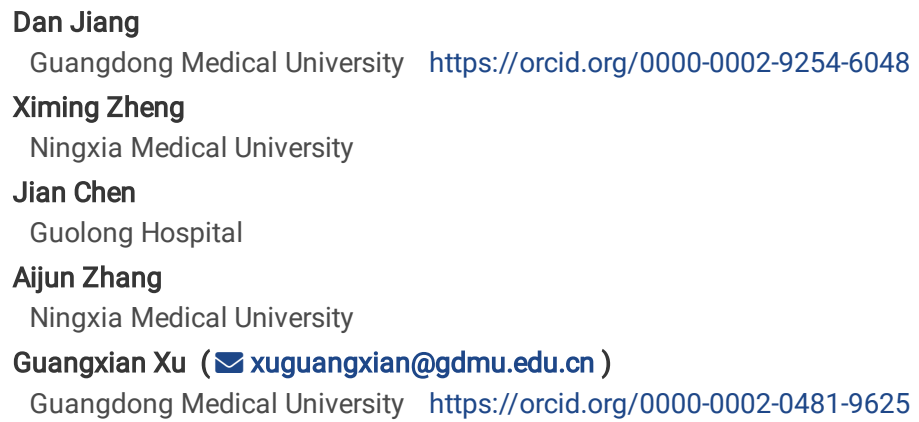

Research article

Keywords: rheumatoid arthritis, microRNA, miR-485-5p, TLR4, IRAK4, diagnostic biomarker

Posted Date: November 10th, 2021

DOI: https://doi.org/10.21203/rs.3.rs-1020256/v1

License: @ (i) This work is licensed under a Creative Commons Attribution 4.0 International License. Read Full License 


\section{Abstract \\ Background}

Rheumatoid arthritis (RA) is a chronic systematic autoimmune disorder that is characterized by symmetrical and inflammatory destruction of distal joints. Dysregulation of microRNAs(miRNAs) are frequently involved in inflammation, and can contribute to pathogenesis and progression of RA. This study aimed to investigate the expression of multiple inflammation-related miRNAs of RA patients and the latent mechanism, and identify novel diagnostic biomarkers.

\section{Methods}

Samples of 100 patients with RA and 72 healthy controls were included. The expression of predicted inflammation-related miRNAs, including miR-16, miR-17, miR-132, miR-140, miR-150, miR-181, miR-200-c, miR-203, miR-223 and miR-485-5p and RA associated genes, including IL-17, IL-18, DAS-28, MMP3, TLR-4, IRAK-4 in plasma and peripheral blood mononuclear cells (PBMCs) of RA patients compared with healthy controls (HC), were detected by qRT-PCR and ELISA. The interaction between miR-485-5p and TLR-4 or IRAK-4 was verified through dual luciferase report assays, western-blot and correlation analysis. The potential of miR-485-5p to be a biomarker for RA diagnostics was valued by ROC curves.

\section{Results}

Among the differentially expressed miRNAs, the expression of miR-485-5p exhibited significantly lower in plasma and PBMCs of the RA patients and was well relevant in the various body fluid samples. The expression of miR-485-5p was negatively correlated with the expression of DAS-28, IL-17, IL-18 and MMP-3, which are significant features of RA. Moreover, the ROC curve of plasma and PBMC miR-485-5p for RA revealed a high diagnostic accuracy. Furthermore, miR485-5p could inhibit the expression of inflammatory cytokines in macrophages by targeting TLR4 and IRAK4, and the expression of miR-485-5p negatively correlated with the level of TLR4 and IRAK4 in the plasma of RA.

\section{Conclusion}

Collectively, our results indicated that down-expression of miR-485-5p was remarkably related to the deterioration of RA progression via the impact on inflammatory cytokines in macrophages, and may serve as a potential diagnostic biomarker and therapeutic target for RA.

\section{Introduction}

Rheumatoid arthritis (RA) is the most common type of inflammatory arthritis with the characteristics of persistent synovitis, systemic inflammation, pain and impaired mobility, affecting approximately 1-3\% of the general population(Scott et al. 2010; Smolen et al. 2016). Genetic and environmental factors are involved in the complex pathogenesis of RA. Numerous evidences proved that inflammation and immunological disorders significantly contribute to development of RA. Proinflammatory cytokines, such as tumor necrosis factor-a (TNF-a), IL-17 and IL-18, have been assigned pivotal roles in the destruction of the joint architecture in patients with RA (Blüml et al. 2014; Furst et al. 2014). And it has been recognized that epigenetic regulation of critical inflammatory factors may be critical but poorly understood in mechanism of RA progression(Salvi et al. 2019).

MicroRNAs (miRNAs) are endogenous small non-coding and evolutionarily conserved single-stranded RNAs (approximately 22 nucleotides) and function predominantly as negative regulators for the expression of target genes by repressing translation or through direct cleavage of mRNA(Lu et al. 2018). In general, miRNAs constitute about $1-2 \%$ of the whole genome and potentially regulate about $30 \%$ of all protein-encoding genes(Kabekkodu et al. 2018 ) .Accumulated evidences proved that miRNAs participated in important cellular processes such as lipid metabolism, differentiation, metabolism, apoptosis, organ development and inflammatory response of immune and non-immune cells(Pers et al. 2013; Rupaimoole et al. 2017; Juźwik et al. 2019). They were also involved in the pathogenesis of various diseases such as cancer, psychiatric and neurological diseases, cardiovascular disease and autoimmune diseases, including RA(Ospelt et al. 2017). MiR-16, miR-21, miR-125b, miR-146a and miR-155 in plasma; miR-24, miR-132, miR-146, miR-150 and miR-155 in PBMCs; or miR-16, miR-155 and miR-223 in synovial fluid of RA patients are upregulated, while miR-23b, miR-30a and miRNA-495 are downregulated in RA(Pauley et al. 2008; Zhu et al. 2012; Filková et al. 2014; Fang et al. 2020; Lv et al. 2021). Dysregulation of miRNAs are closely related to inflammatory pathways, proinflammatory cytokines expression and other processes involved in RA(Evangelatos et al. 2019). In addition, miRNAs in circulations are wellprotected from endogenous RNase activity and exhibit high stability in the cell free fluids, making them novel candidate biomarkers for diagnosis and prognosis of diseases(Backes et al. 2016). As a result, identifying inflammation related miRNAs in the circulation of RA patients and analyzing the roles of these miRNAs in inflammatory reaction may provide new sight into the mechanism of RA development, as well as diagnostic and therapeutic strategy for RA.

In the present study, we investigated the expression of several inflammation-related miRNAs in plasma and PBMCs of RA patients, and the correlation between miRNAs and disease activities of RA. Additionally, the impact of miR-485-5p, one of the differentially expressed inflammation-related miRNAs, on the expression of inflammatory cytokines in macrophages and the molecular mechanism were also analyzed. Collectively, the results indicated the critical roles of miR-485-5p serving as an effector on the expression of inflammatory cytokines in macrophages and RA, and implicate the potential application of miR-485-5p in RA diagnosis.

\section{Materials And Methods}


172 subjects including100 RA patients (RA) and 72 healthy controls (HC) were recruited from General Hospital of Ningxia Medical University. Clinical features of the participants were shown in table 1. RA group were diagnosed according to the criteria of the American College of Rheumatology, and $\mathrm{HC}$ group were through the same period health examination and without a history of knee surgery, or keen degeneration disease a, or inflammatory diseases. The selected subjects were excluded from other autoimmune diseases and major organ system disease. Both plasma and PBMCs were obtained from the two groups patients. This study was approved by the General Hospital of Ningxia Medical University Ethics Committee and is in compliance with the Helsinki Declaration. Informed consent was obtained from the patients.

\section{Preparation of plasma and PBMC}

Blood samples $(5 \mathrm{ml})$ were collected with ethylene diamine tetraacetic acid (EDTA-2K) containing tube, and plasma was separated by centrifugation at $1600 \mathrm{~g}$ for $10 \mathrm{~min}$ at room temperature $₫$ followed by centrifugation at $16,000 \mathrm{~g}$ for $10 \mathrm{~min}$ at $4^{\circ} \mathrm{C}$ to remove all cell debris. The plasma supernatant was collected and

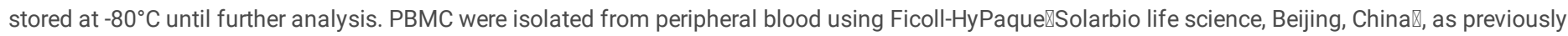
described(Pham et al. 2008). Briefly, blood was collected into $10 \mathrm{~mL}$ tubes containing heparin as anticoagulant. Immediately after collection, the cells were separated from whole blood by centrifugation on a Ficoll-Hypaque density gradient. The PBMC were recovered and washed three times with $1 \mathrm{~mL}$ RPMI culture medium, and adjusted to a final concentration of $1 \times 10^{7}$ cells $/ \mathrm{mL}$. The PBMC pellet was stored at $-80^{\circ} \mathrm{C}$ until further analysis.

\section{RNA isolation, reverse transcription and quantitative PCR}

RNA was isolated using RNAiso Plus isolation kit (TaKaRa, Dalian, China) according to manufacturer`s protocol. Nanodrop 2000(Thermo Fisher Scientific, CA, USA) was used to assess the concentration and purification of RNA. Reverse transcription was performed using Revert aid first strand cDNA sythesis kit (Thermo Scientific, CA, USA) with the corresponding primers $\left(42^{\circ} \mathrm{C}\right.$ for $15 \mathrm{~min}, 70^{\circ} \mathrm{C}$ for $\left.5 \mathrm{~min}\right)$. For constructing cDNA standard curves of miRNAs and U6, the target sequences were amplified using 2×Ecotap PCR SuperMix (TransGen Biotech Co., Ltd., Beijing, China) with specific primers under the following conditions:and cloned into pEASY-T1 Cloning vector (TransGen Biotech Co., Ltd., Beijing, China), the concentrations of plasmid were detected using Nanodrop 2000 (Thermo Fisher Scientific, CA, USA), then the plasmids were diluted in series by ten times to $10^{-8}$ and finally the standard curve was obtained by determining the corresponding threshold cycle (Ct), respectively. For detecting the expression of miRNAs, qRT-PCR was performed using SYBR select master mix ( $\mathrm{ABI}$, Carlsbad, $\mathrm{CA}, \mathrm{USA}$ ) on $\mathrm{ABI}$ StepOnePlus qPCR system ( $\mathrm{ABI}$, Carlsbad, $\mathrm{CA}, \mathrm{USA})$ under the following conditions: $95^{\circ} \mathrm{C}$ for 5 min followed by $40 \mathrm{cycles}$ consisting of $95^{\circ} \mathrm{C}$ for $5 \mathrm{sec}, 62^{\circ} \mathrm{C}$ for $30 \mathrm{sec}$ and $72{ }^{\circ} \mathrm{C}$ for $30 \mathrm{sec}$. The experiment was repeated three times. The copy numbers of experimental RNAs were calculated after real-time amplification from the linear regression of that standard curve. The relative expression of miRNAs was calculated using $2^{-\Delta \Delta C t}$ method and U6 snRNA was used for normalization. The primers specific for the target genes were designed using bioinformatics tools, synthesized in Sangon Biotech Inc. (Shanghai, China).

Primer sequences were as follows: for miR-16, 5- TAGCAGCACGT $\forall A T A T$ - 3; for miR-17, 5- $C \forall A G T G C$ T ACAGTGCA - 3; for miR-132, 5 - $T \forall C A G T C T A C A G \mathbb{C} A T G$ - 3; for miR-140, 5- AGTGG T T ACCTATG - 3; for miR-150, 5- TCTCC $\forall \mathbb{C} C$ T GTAC - 3; for miR-181a, 5 - $\forall C A T C \forall C G C T G T C G G$ - 3; for miR-200c, 5- T $\forall T A C T G \mathbb{C} G G G T \forall T G$ - 3; for miR-203, 5- TG $\forall A T G$ T TAGGACA - 3; for miR-223, 5 - TGTCAG T TGTC $\forall A T A C$ - 3; for miR-485-5p, 5- AGAGGCTGGC GTGAT - 3; the universal antisense primer, 5- $C T C \forall C T G G T G T C G T G G A$ - 3; for U6, sense 5- CTCGC Т CGGCAGCACA - 3, antisense 5- CGC Т CACG $\forall$ Т TGCGT - 3.

\section{Cells and cell culture}

Murine macrophage cell line (RAW264.7) and human embryonic cell line (HEK-293T) were purchased from the Institute of Biochemistry and Cell Biology (Chinese Academy of Sciences, Shanghai, China). Cells were collected and cultivated at $37^{\circ} \mathrm{C}$ with $5 \% \mathrm{CO}_{2}$ in DMEM high glucose medium supplemented with $10 \%$ fetal bovine serum (FBS), $100 \mathrm{U} / \mathrm{mL}$ penicillin and $100 \mu \mathrm{g} / \mathrm{mL}$ streptomycin (GIBCO, Invitrogen, USA).

\section{Cell transfection}

RAW264.7 cells were transiently transfected with 50 nM miR-485-5p control or miR-485-5p mimic (GenePharma, Shanghai, China); 50 nM control or miR-485$5 p$ inhibitor (GenePharma, Shanghai, China), using Lipofectamine 2000 (Invitrogen, USA) according to the manufacturer`s instructions.

\section{Plasmid construction and dual luciferase reporter assay}

To validate the targeting role of miR-485-5p to TLR4 and IRAK4, the wild-type of TLR4 and IRAK-4 3 UTRs, whichconta $\in$ the $b \in d \in$ ge $\leq$ mentsofmiR $-485-5 p$, wereobta $\in$ edbyPCR. Thenthe 4 tant3UTRs of TLR-4 and IRAK-4 were obtained by site-directed mutagenesis. The primers used during the study were as follows: wild-type TLR-4 3UTR(F or ward, 5-CCCAAGCTTCACTGCCAGGAGAACTA-3 ; Reverse, 5-CGGACTAGTTCATAACGGCTACACCA-3), $\mu \tan t T L R$ - 43UTR (Forward, 5- C $C \forall G C$ T CACTGC AGGAG $\forall C T A$ - 3; Reverse, 5 - CGGACTAGTGCGACAGATG $\forall C A$ T - 3), wild-type IRAK-4 3UTR(F or ward, 5-CCCAAGCTTTTACTTATTGCCTGTACC-3; Reverse, 5CGGACTAGTATAGTTAGCCCGTTTTG-3), $\mu \tan t I R A K$ - 43UTR (Forward, 5- $C$ C $\forall G C$ T CACTGC AGGAG $\forall C T A$ - 3; Reverse, 5

- CGGACTAGTGCGACGTGGT $\forall \mathrm{T} T A T$ - 3). These above-mentioned wild-type or mutated 3'UTR fragments were then subcloned into pMIR-Report vector (Promega, Madison, WI, USA).

HEK-293T cells were plated into 96-well plates and co-transfected with 120ng of either pMIR-TLR4-WT/ TLR4-Mut/ IRAK4-WT / IRAK4-Mut, 3 pmol miR-485-5p mimic, inhibitor, nc囚the control comparing with mimic or inhibitor》 and 80ng PRL-TK internal-plasmid using Lipofectamine 2000 (Invitrogen, Carlsbad, CA, USA). The relative luciferase activity at $48 \mathrm{~h}$ post-transfection was determined by the Dual-Luciferase Reporter Assay System (Promega, Madison, WI, USA) following the manufacturer's protocol. Transfections were done in triplicate and repeated at least thrice in independent experiments.

Processing math: $100 \%$ 


\section{Western-Blot Analysis}

After extensively washing, cells were lysed using Whole Cell Lysis Assay and proteins were harvested according to the protocol (KeyGen Biotech Inc., Nanjing, China). Protein concentrations was determined by the BCA Assay kit ((KeyGen Biotech Inc., Nanjing, China). Total protein of each lysate was then separated by SDS-PAGE and subsequently transferred onto PVDF membranes (Millipore, MA, USA). Afterwards, the membranes were blocked with $5 \%$ non-fat milk in TBST at room temperature for $1 \mathrm{~h}$, followed by incubated with antibodies against TLR4 (1:2,000, cat. no. 14358, Cell

Signaling Technology, Inc), or IRAK4 (1:2000, cat. no. 4363, cell Signaling Technoloy, Inc), or GAPDH (1:2,000, ab8245, Abcam) overnight at $4{ }^{\circ} \mathrm{C}$. After washing with TBST, membranes were striped with appropriate HRP-conjugated secondary antibodies for $1 \mathrm{~h}$ at room temperature, followed by visualized using the Luminol reagent (Thermo Fisher Scientific, Carlsbad, CA, USA). Eventually, bands were imaged and densitometric analyzed by a chemiluminescence detection system (Bio-Rad, USA).

\section{Enzyme linked immunosorbent assay (ELISA)}

The levels of IL-17, IL-18, DAS-28, MMP3, TLR-4, IRAK-4 in the peripheral blood and PBMC cells of patients and were detected using ELISA kits (Bangyi Biotech, Shanghai, China) according to the manufacturer`s protocol.

\section{Bioinformatics analysis}

MiRWalk (http://mirwalk.umm.uni-heidelberg.de/),miRanda (http://www.microRNA.org), TargetScan (http://www.targetscan.org) and DIANA LAB (http://83.212.96.7/DianaToolsNew/index.php?r=mirpath) were used to predict and select miRNAs associated with inflammation, and the predicted relationship were analyzed by Cytoscape.

\section{Statistical analysis}

Data were presented as the mean \pm standard deviation. Statistical analyses were performed using SPSS 17.0 (IBM, CA, USA). Differences between two groups were analyzed with Student`s $t$-test. Differences among three groups were analyzed with Analysis of One-way ANOVA. Correlations with miRNA concentrations and other clinical factors were analyzed with Pearson product-moment correlation coefficient. A $P$ - value less than 0.05 was considered statistically significant.

\section{Results}

\section{The differentially expressed miRNAs in plasma and PBMC of RA and HC}

Undoubtedly, the inflammatory factors are main effectors in the development of RA, and many reports indicated miRNAs could impress the progression of RA via targeting these factors(Evangelatos et al. 2019). In order to study the roles of miRNAs associated with these inflammatory factors in RA, we predicted the possible miRNAs through bioinformatical analysis, and miR-16, miR-17, miR-132, miR-140, miR-150, miR-181, miR-200c, miR-203, miR-223 and miR-485-5p were found to may be able to affect the development of RA via regulating the expressions of various inflammatory factors (Figure 1). To determine whether miRNAs can be used as diagnostic biomarkers for patients with RA, the expression of miR-16, miR-17, miR-132, miR -140, miR-150, miR-181, miR-200c, miR203, miR-223 and miR-485-5p in plasma and PBMC of RA patients and HC were analyzed.

As shown in table 2, in plasma, miR-16, miR-17, miR-140, miR-150, miR-200c, miR-203 and miR-223 of patients with RA were significantly higher than those of $\mathrm{HC}$, while the miR-485-5p and miR-132 were significant lower in RA than that of HC. In PBMC, only miR-485-5p was significant lower in RA than that of HC, while other nine of the detected miRNAs increased in RA compared to HC

Consistently, there were previous reports that showed the levels of miR-16, miR-17, miR-132, miR-140, miR-150, miR-200c, miR-203 and miR-223 were changed in RA. But there was no report for miR-485-5p associated with RA. Additionally, our results indicated that miR-485-5p decreased both in plasma and PBMC in $\mathrm{RA}$ in comparison with $\mathrm{HC}$, indicating that miR-485-5p maybe a potential diagnostic biomarker for patients with RA and making it become the focus of our study.

\section{The potential of miR-485-5p of plasma and PBMC as diagnostic markers of RA.}

To assess the possibility of miR-485-5p as a biomarker of RA, we investigated the correlation of expression of miR-485-5p and other clinical variables including 28-joint Disease Activity Score (DAS28), IL-17, IL-18 and metalloproteinase-3 (MMP-3). The results indicated that expression of miR-485-5p in plasma and PBMC of RA patients remarkably inverse correlated with DAS28 ( $r=-0.845, p=0.008, n=30 ; r=-0.866, p<0.001$, $n=30 ;$ respectively ), IL-17 ( $r=$ $-0.667, p<0.001, n=30 ; r=-0.680, p<0.001, n=30$; respectively ), IL-18 ( $=-0.877, p<0.001, n=30 ; r=-0.903, p<0.001, n=30 ;$ respectively) and MMP-3 ( $r=$ $-0.667, \mathrm{p}<0.001, \mathrm{n}=30 ; \mathrm{r}=-0.683, \mathrm{p}<0.001, \mathrm{n}=30$; respectively) (Figure 2).

To determine the diagnostic ability of miR-485-5p of plasma and PBMC for patients with RA, we conducted ROC analysis. MiR-485-5p of plasma at a cut off value of 319.8 copes/ $\mu \mathrm{L}$ could detect individuals with RA at $97 \%$ of sensitivity and $97.2 \%$ of specificity. MiR-485-5p of PBMC at a cutoff value of 609.1 copes/ $\mu \mathrm{L}$ could detect individuals with RA at $98 \%$ of sensitivity and $97.2 \%$ of specificity. Besides, the area under the ROC curve (AUC) was not less than 0.90 , indicating that the expression of miR-485-5p in plasma and PBMC exhibit high diagnostic capability (Figure 3).

miR-485-5p suppresses TLR-4 and IRAK-4 by interacting with their 3` UTR 
Given the great potential of miR-485-5p for diagnosis of RA, the latent mechanism was furtherly studied. Firstly, as shown in table 2, miR-485-5p of plasma and PBMC were significantly down-expressed in RA relative to HC. Then, bioinformatical analysis indicated that it was involved in various pathways affecting the progression of diseases, including inflammatory reaction (Figure 4A). Furthermore, prediction by miRWalk, miRDB, miRanda, and Targetscan testified that TLR-4 and IRAK4 who were important components of inflammatory pathway were showed to be potential targets of miR-485-5p.

Sequence alignment indicated that miR-485-5p was matched with 3

UTRofTLR - 4 and IRAK- 4 and $f$ or detect $\in$ gtheco $\cap$ ectionbetweenmiR - 485 - $5 p$ and TLR - 4 and IRAK, thetar $\geq t \in g s i t e w e r e \mu t a t e d$;

UTR binding sites.

\section{miR-485-5p inhibits the expression of inflammatory cytokines in macrophages.}

TLR4 and IRAK4 are identified as critical signaling molecules of TLRs/ NF-KB pathway and can activate NF-kB pathway to regulate inflammatory reaction through accumulating kinds of signaling molecules. To determine the effect of miR-485-5p on the downstream signals of TLR4 and IRAK4, we tested the expression of IL-6, IL-17, IL-18 and MMP-3 after transfection of miR-485-5p mimics, inhibitor and their comparative controls in macrophage. The results indicated that overexpression of miR-485-5p significantly decreased the release of IL-6, IL-17, IL-18 and MMP-3 in macrophages, and vice versa (Figure5).

\section{miR-485-5p negatively correlates with TLR-4 and IRAK-4 in plasma of RA patients.}

Then, to determine the correlation between the level of miR-485-5p and TLR-4 or IRAK4 in plasma of RA patients, we detected TLR4 and IRAK4 level in plasma of RA. The results indicated that the expression of TLR-4 (Figure 6A) and IRAK-4 (Figure 6B) were significantly increased in plasma of RA patient compared to that of HC. And correlation analysis showed that miR-485-5p in plasma was respectively negatively correlated with TLR-4 ( $r=-0.937, p<0.001)(F i g u r e ~ 6 C)$ and IRAK-4 ( $r=-0.748, p<0.001$ ) (Figure 6D). These data suggested that low expression of miR-485-5p presented a significant negative correlation with high expression of TLR4 and IRAK4 in patients with RA. These results further indicated that miR-485-5p's impression on RA is associated with dysregulation of TLA-4 and IRAK-4 A and provided the basis for studying the probability of miR-485-5p to be a biomarker of RA.

\section{Discussion}

MicroRNAs are short, conserved, noncoding RNA molecules participated in fundamental biological process including apoptosis, proliferation, and metabolism of glucose and lipids by regulating gene expression at transcriptional and translational levels. Besides as regulators of gene expression, miRNAs were recently noted as predictive biomarkers for various pathological conditions(Liu et al. 2014). MiRNAs are remarkable stable in plasma or other body fluid as they were protected from endogenous RNase activity(Mitchell et al. 2008). Previous researches indicated that the plasma or synovial fluid miRNAs are stable at room temperature up to $24 \mathrm{~h}$ or $-20^{\circ} \mathrm{C}$ up to seven days and resistant for freeze-thawing from $-80^{\circ} \mathrm{C}$ to room temperature up to several times. These excellent stabilities enable miRNAs to be the most suitable candidate for disease diagnostic(Murata et al. 2010). Therefore, plasma miRNAs can be diagnostic biomarkers of various carcinoma, neurodegenerative and inflammatory diseases(Backes et al. 2016). In this study, we investigated the expression of miRNAs in plasma and PBMC of patients with RA to determine whether the specific miRNAs could be used as biomarker for RA.

Ten miRNAs associated with inflammatory responses, including miR-16, miR-17, miR-132, miR-140, miR-150, miR-181, miR-200c, miR-203, miR-223 and miR485-5p of plasma and PBMC of RA patients and HCs were quantified by RT-PCR. The results indicated that only miR-485-5p was significantly down-regulated, while other nine miRNAs increased in the patients with RA. Consistent with previous reports, miR-132 was involved in systemic processes as a consequence of joint inflammation and correlated inversely with TJC in RA(Nakasa et al. 2008). The miR-200c, associating with regulation of epithelial to mesenchymal transition (EMT) and metastasis, has been showed to up-regulated in various cancers and was considered to be a valuable biomarker of these malignant cancers(Mutlu et al. 2016; Byun et al. 2019). MiR-203, with the ability of stimulating the expression of matrix metalloproteinase-1 (MMP-1) and proinflammatory cytokines, was significant up-regulated in RA when compared to that of HC(Stanczyk et al. 2011). MiR-223 plays an important role in the differentiation of osteoclasts, which are critical for the joint state in RA patients. Numerous researches indicated that miR-223 was up-regulated within sites of inflammation as well as in peripheral circulation in patients with RA(Chen 2014; Taha et al. 2020). Our results also showed the level of miR-223 was significantly increased in the body fluid of patients with RA in comparison to HC. However, researches have revealed the level of miR-223 did not correlate with age, DAS28, CRP, ACPAs, or with disease duration, indicating that miR-223 may not be suitable for the diagnostics of RA (Fulci et al. 2010).

MiR-485-5p has been reported to be significantly down-regulated in various human cancers and has potential to be as diagnostic biomarker of these cancers(Lou et al. 2016; Wang et al. 2018; Wang et al. 2020). Our study firstly reported that miR-485-5p had distinguishable expression between RA and HC. The expression of miR-485-5p in plasma and PBMC of patients with RA were remarkable lower than that of HC. The correlation analysis between clinical variables of RA and the expression of miR-485-5p indicated that miR-485-5p in plasma and PBMC was consistently correlated to DAS28, IL-17, IL-18 and MMP-3 with a reverse manner. Subsequently, ROC curves were used to estimate the sensitivity and specificity of miR-485-5p as diagnostic markers for RA. The AUCs of plasma and PBMC miR-485-5p for RA reached to greater than 0.97, which were high enough for miR-485-5p to be accurate for RA diagnostics.

Recent study showed that miR-485-5p was involved in the TNF-a induced TRADD signaling pathway(Chen et al. 2016). The pro-inflammatory cytokines, such as TNF- $\alpha$ and IL-1 $\beta$ induced activation of NF-KB signals play pivotal role in regulating the inflammatory response in RA(Chen et al. 2016; Friedman et al. 2019; Jimi et al. 2019). MiR-20a, miR-146a and miR-155 were revealed to inhibit inflammatory response and reduce MMPs synthesis and joint damage by regulating NF-KB signaling pathways(Zhou et al. 2015; Xie et al. 2020). TLR4 stimulation in human synovial fibroblasts leads to NF-kB activation and cytokines release and contributes to the pathogenesis of RA(Goh et al. 2012). Meanwhile, the targets of miR-485-5p were predicted and verified in this study, and the results revealed that TLR4 and IRAK4 were possible targets of miR-485-3p. Overall, miR-485-5p may play roles in the development of RA via targeting TLR4 and IRAK4 and reaulatina the associated inflammation responses.

Processing math: $100 \%$ 


\section{Conclusion}

In conclusion, we revealed the lower expression of miR-485-5p in plasma and PBMC of patients with RA in comparison with HC, and found the decrease of miR-485-5p was remarkably related to the deterioration of RA progression. Meanwhile, through ROC curve analysis, miR-485-5p was verified that has high sensitivity and specificity to be a diagnostic biomarker of RA. In addition, miR-485-5p was found that can regulate inflammation response by targeting TLR4 and IRAK4. Taken together, miR-485-5p might be as a potential diagnostic biomarker and therapeutic target for RA.

\section{Abbreviations}

DAS-28: Disease activity score for 28 joints; ELISA: enzyme linked immunosorbent assay; HC: healthy controls; IL-17: Interleukin-17; IL-18: Interleukin-18; IRAK4: interleukin-1 receptor-associated kinase 4; miRNA: microRNA; MMP-3: matrix metalloproteinase 3; mut: mutation囚nc: normal control; PBMCs: peripheral blood mononuclear cells; qRT-PCR: quantitative Real-time PCR; RA: rheumatoid arthritis; TLR-4: Toll-like receptor 4; TNF-a: tumor necrosis factor-a; GAPDH: glyceraldehyde-3-phosphate dehydrogenase; WT囚wild type区

\section{Declarations}

\section{Acknowledgements}

The authors thank all patients and healthy volunteers that participated in the study.

\section{Authors' contributions}

JD, ZX and XG analyzed and interpreted the patient data and wrote the manuscript. JD, XG, ZX, ZA and CJ collected materials and performed the experiments. All authors read and approved the final manuscript.

\section{Funding}

The study was partially supported by discipline construction project of Guangdong Medical University (No.4SG21278P).

\section{Availability of data and materials}

All data generated or analyzed during this study are included in this published article.

\section{Ethics approval and consent to participate}

All participants provided informed written consent for participation in the study. The study protocol and informed consent were approved by the General Hospital of Ningxia Medical University Ethics Committee and conformed to the ethical guidelines of the Helsinki Declaration.

\section{Consent for publication}

Not-applicable.

\section{Competing interests}

The authors declare that they have no competing interests.

\section{References}

1. Backes, C, Meese, E, and Keller, A. Specific miRNA Disease Biomarkers in Blood, Serum and Plasma: Challenges and Prospects. Mol Diagn Ther. 2016; 20:509-518.

2. Blüml, S, Redlich, K, and Smolen, JS. Mechanisms of tissue damage in arthritis. Semin Immunopathol. 2014; 36:531-540.

3. Byun, Y, Choi, YC, Jeong, Y, Lee, G, Yoon, S, Jeong, Y, Yoon, J, and Baek, K. MiR-200c downregulates HIF-1a and inhibits migration of lung cancer cells. Cell Mol Biol Lett. 2019; 24:28.

4. Chen, SY. MicroRNA-223: a double-edged sword in rheumatoid arthritis. Rheumatol Int. 2014; 34:285-286.

5. Chen, Z, Zhang, Z, Zhang, D, Li, H, and Sun, Z. Hydrogen sulfide protects against TNF-a induced neuronal cell apoptosis through miR-485-5p/TRADD signaling. Biochem Biophys Res Commun. 2016; 478:1304-1309.

6. Evangelatos, G, Fragoulis, GE, Koulouri, V, and Lambrou, GI. MicroRNAs in rheumatoid arthritis: From pathogenesis to clinical impact. Autoimmun Rev. 2019; 18:102391.

7. Fang, L, Xu, XF, Lu, Y, Wu, YY, and Li, JJ. MicroRNA-495 attenuates proliferation and inflammatory response in rheumatoid arthritis fibroblast-like synoviocytes through attenuating ß-catenin pathway. J Biol Regul Homeost Agents. 2020; 34:837-844.

8. Filková, M, Aradi, B, Senolt, L, Ospelt, C, Vettori, S, Mann, H, Filer, A, Raza, K, Buckley, CD, Snow, M, Vencovský, J, Pavelka, K, Michel, BA, Gay, RE, Gay, S, and Jüngel, A. Association of circulating miR-223 and miR-16 with disease activity in patients with early rheumatoid arthritis. Ann Rheum Dis. 2014; 73:18981904.

a Friedman B and Cronstein, B. Methotrexate mechanism in treatment of rheumatoid arthritis. Joint Bone Spine. 2019; 86:301-307.

Processing math: $100 \%$ 
10. Fulci, V, Scappucci, G, Sebastiani, GD, Giannitti, C, Franceschini, D, Meloni, F, Colombo, T, Citarella, F, Barnaba, V, Minisola, G, Galeazzi, M, and Macino, G. miR-223 is overexpressed in T-lymphocytes of patients affected by rheumatoid arthritis. Hum Immunol. 2010; 71:206-211.

11. Furst, DE, and Emery, P. Rheumatoid arthritis pathophysiology: update on emerging cytokine and cytokine-associated cell targets. Rheumatology (Oxford). 2014; 53:1560-1569.

12. Goh, FG, and Midwood, KS. Intrinsic danger: activation of Toll-like receptors in rheumatoid arthritis. Rheumatology (Oxford). 2012; 51:7-23.

13. Jimi, E, Fei, H, and Nakatomi, C. NF-kB Signaling Regulates Physiological and Pathological Chondrogenesis. Int J Mol Sci. 2019; 20:

14. Juźwik, CA, S, SD, Zhang, Y, Paradis-Isler, N, Sylvester, A, Amar-Zifkin, A, Douglas, C, Morquette, B, Moore, CS, and Fournier, AE. microRNA dysregulation in neurodegenerative diseases: A systematic review. Prog Neurobiol. 2019; 182:101664.

15. Kabekkodu, SP, Shukla, V, Varghese, VK, J, DS, Chakrabarty, S, and Satyamoorthy, K. Clustered miRNAs and their role in biological functions and diseases. Biol Rev Camb Philos Soc. 2018; 93:1955-1986.

16. Liu, B, Li, J, and Cairns, MJ. Identifying miRNAs, targets and functions. Brief Bioinform. 2014; 15:1-19.

17. Lou, C, Xiao, M, Cheng, S, Lu, X, Jia, S, Ren, Y, and Li, Z. MiR-485-3p and miR-485-5p suppress breast cancer cell metastasis by inhibiting PGC-1a expression. Cell Death Dis. 2016; 7:e2159.

18. Lu, TX, and Rothenberg, ME. MicroRNA. J Allergy Clin Immunol. 2018; 141:1202-1207.

19. Lv, X, Huang, J, and Wang, H. MiR-30a-3p ameliorates oxidative stress in rheumatoid arthritis synovial fibroblasts via activation of Nrf2-ARE signaling pathway. Immunol Lett. 2021; 232:1-8.

20. Mitchell, PS, Parkin, RK, Kroh, EM, Fritz, BR, Wyman, SK, Pogosova-Agadjanyan, EL, Peterson, A, Noteboom, J, O'Briant, KC, Allen, A, Lin, DW, Urban, N, Drescher, CW, Knudsen, BS, Stirewalt, DL, Gentleman, R, Vessella, RL, Nelson, PS, Martin, DB, and Tewari, M. Circulating microRNAs as stable blood-based markers for cancer detection. Proc Natl Acad Sci U S A. 2008; 105:10513-10518.

21. Murata, K, Yoshitomi, H, Tanida, S, Ishikawa, M, Nishitani, K, Ito, H, and Nakamura, T. Plasma and synovial fluid microRNAs as potential biomarkers of rheumatoid arthritis and osteoarthritis. Arthritis Res Ther. 2010; 12:R86.

22. Mutlu, M, Raza, U, Saatci, Ö, Eyüpoğlu, E, Yurdusev, E, and Şahin, Ö. miR-200c: a versatile watchdog in cancer progression, EMT, and drug resistance. J Mol Med (Berl). 2016; 94:629-644.

23. Nakasa, T, Miyaki, S, Okubo, A, Hashimoto, M, Nishida, K, Ochi, M, and Asahara, H. Expression of microRNA-146 in rheumatoid arthritis synovial tissue. Arthritis Rheum. 2008; 58:1284-1292.

24. Ospelt, C, Gay, S, and Klein, K. Epigenetics in the pathogenesis of RA. Semin Immunopathol. 2017; 39:409-419.

25. Pauley, KM, Satoh, M, Chan, AL, Bubb, MR, Reeves, WH, and Chan, EK. Upregulated miR-146a expression in peripheral blood mononuclear cells from rheumatoid arthritis patients. Arthritis Res Ther. 2008; 10:R101.

26. Pers, YM, and Jorgensen, C. MicroRNA in 2012: Biotherapeutic potential of microRNAs in rheumatic diseases. Nat Rev Rheumatol. 2013; 9:76-78.

27. Pham, TN, King, D, Macparland, SA, McGrath, JS, Reddy, SB, Bursey, FR, and Michalak, TI. Hepatitis C virus replicates in the same immune cell subsets in chronic hepatitis $C$ and occult infection. Gastroenterology. 2008; 134:812-822.

28. Rupaimoole, R, and Slack, FJ. MicroRNA therapeutics: towards a new era for the management of cancer and other diseases. Nat Rev Drug Discov. 2017; 16:203-222.

29. Salvi, V, Gianello, V, Tiberio, L, Sozzani, S, and Bosisio, D. Cytokine Targeting by miRNAs in Autoimmune Diseases. Front Immunol. $2019 ; 10: 15$.

30. Scott, DL, Wolfe, F, and Huizinga, TW. Rheumatoid arthritis. Lancet. 2010; 376:1094-1108.

31. Smolen, JS, Aletaha, D, and Mclnnes, IB. Rheumatoid arthritis. Lancet. 2016; 388:2023-2038.

32. Stanczyk, J, Ospelt, C, Karouzakis, E, Filer, A, Raza, K, Kolling, C, Gay, R, Buckley, CD, Tak, PP, Gay, S, and Kyburz, D. Altered expression of microRNA-203 in rheumatoid arthritis synovial fibroblasts and its role in fibroblast activation. Arthritis Rheum. 2011; 63:373-381.

33. Taha, M, Shaker, OG, Abdelsalam, E, and Taha, N. Serum a proliferation-inducing ligand and MicroRNA-223 are associated with rheumatoid arthritis: diagnostic and prognostic implications. Mol Med. 2020; 26:92.

34. Wang, FR, Xu, SH, Wang, BM, and Wang, F. MiR-485-5p inhibits metastasis and proliferation of osteosarcoma by targeting CX3CL1. Eur Rev Med Pharmacol Sci. 2018; 22:7197-7204.

35. Wang, X, Zhou, X, Zeng, F, Wu, X, and Li, H. miR-485-5p inhibits the progression of breast cancer cells by negatively regulating MUC1. Breast Cancer. 2020; 27:765-775.

36. Xie, Z, Shen, P, Qu, Y, Xu, J, Zheng, C, Gao, Y, and Wang, B. MiR-20a inhibits the progression of human arthritis fibroblast-like synoviocytes and inflammatory factor expression by targeting ADAM10. Environ Toxicol. 2020; 35:867-878.

37. Zhou, Q, Haupt, S, Kreuzer, JT, Hammitzsch, A, Proft, F, Neumann, C, Leipe, J, Witt, M, Schulze-Koops, H, and Skapenko, A. Decreased expression of miR146a and miR-155 contributes to an abnormal Treg phenotype in patients with rheumatoid arthritis. Ann Rheum Dis. 2015; 74:1265-1274.

38. Zhu, S, Pan, W, Song, X, Liu, Y, Shao, X, Tang, Y, Liang, D, He, D, Wang, H, Liu, W, Shi, Y, Harley, JB, Shen, N, and Qian, Y. The microRNA miR-23b suppresses IL-17-associated autoimmune inflammation by targeting TAB2, TAB3 and IKK-a. Nat Med. 2012; 18:1077-1086.

\section{Tables}

Table 1: Clinical features of the participants

Processing math: $100 \%$ 


\begin{tabular}{|lll|}
\hline Characteristics & RA & HC \\
\hline Number of participants & 100 & 72 \\
\hline Sex, male/female & $25 / 75$ & $28 / 44$ \\
\hline Age, mean (range) & $46.6(21$ to 75$)$ & $41.6(23$ to 70$)$ \\
\hline Disease duration (y), (range) & $14.5(0.2$ to 42$)$ & NA \\
\hline Positive RF, n (\%) & $79(79.0)$ & NA \\
\hline Positive ACCP antibody,n (\%) & $71(71.0)$ & NA \\
\hline Positive ASO, n (\%) & $43(43.0)$ & NA \\
\hline ESR (mm), mean (range) & $33.5(2$ to 104$)$ & NA \\
\hline CRP (mg/dl), mean (range) & $26.2(2.7$ to 70.5$)$ & NA \\
\hline IL-17(ng/ml), mean (range) & $327.4(238.5$ to 443.3) & NA \\
\hline IL-18(ng/ml), mean (range) & $167.2(96.2$ to 229.9$)$ & NA \\
\hline MMP3 (ng/ml), mean (range) & $324.9(238.3$ to 427.3) & NA \\
\hline DAS28, mean (range) & $4.1(2.3$ to 6.7) & NA \\
\hline SJC, mean (range) & $2.6(0$ to 11$)$ & NA \\
\hline TJC, mean (range) & $3.9(0$ to 15$)$ & NA \\
\hline VAS, mean (range) & $45.9(10$ to 85$)$ & NA \\
\hline
\end{tabular}

RF, Rheumatoid factor; ACCP, anti-cyclic citrullinated peptide; ASO, antistreptolysin 0 test; CRP, C-reactive protein; ESR, erythrocyte sedimentaition ratio; MMP-3, metalloproteinase-3; DAS28, 28-joint Disease Activity Score; SJC, swollen joint count; TJC, tender joint count; VAS, visual analogue scale of general health; RA, rheumatoid arthritis; $\mathrm{HC}$, healthy control; NA, not applicable.

Table 2 The expression of miRNAs in plasma and PBMC of RA and HC

\begin{tabular}{|lllll|}
\hline Gene & RA & \multicolumn{3}{c|}{ HC } \\
\cline { 2 - 5 } & Plasma & PBMC & Plasma & PBMC \\
\hline miR-16 & $1.44 * 1010 \Delta$ & $6.24 * 1011+$ & $2.11 * 109$ & $2.07 * 1011$ \\
\hline miR-17 & $4.89 * 103 \Delta$ & $7.50 * 103+$ & $1.54 * 103$ & $1.88 * 103$ \\
\hline miR-132 & $215.00 \#$ & $6.32 * 102+$ & 940.30 & 237.08 \\
\hline miR-140 & $1.17 * 1011 \Delta$ & $3.32 * 109+$ & $2.86 * 1010$ & $2.52 * 108$ \\
\hline miR-150 & $3.60 * 105 \Delta$ & $1.61 * 104+$ & $1.61 * 105$ & $3.80 * 103$ \\
\hline miR-181 & $2.11 * 104 \Delta$ & $626.61+$ & $5.09 * 103$ & 235.90 \\
\hline miR-200c & $665.92 \Delta$ & $2.97 * 104+$ & 180.45 & $8.55 * 103$ \\
\hline miR-203 & $4.86 * 103 \Delta$ & $561.49+$ & $1.61 * 103$ & 167.58 \\
\hline miR-223 & $4.33 * 103 \Delta$ & $6.76 * 104+$ & $2.39 * 103$ & $2.12 * 104$ \\
\hline miR-485-5p & $138.27 \#$ & $270.16-$ & 617.50 & 924.60 \\
\hline
\end{tabular}

Note:

$\Delta$ Relative to HC, RA increased expression levels of miRNAs in plasma, $\mathrm{p}<0.05$;

\# Relative to HC, RA decreased expression levels of miRNAs in plasma, $p<0.05$;

+ Relative to HC, RA increased expression levels of miRNAs in PBMC, $p<0.05$;

- Relative to HC, RA decreased expression levels of miRNAs in PBMC, $p<0.05$;

\section{Figures}


A

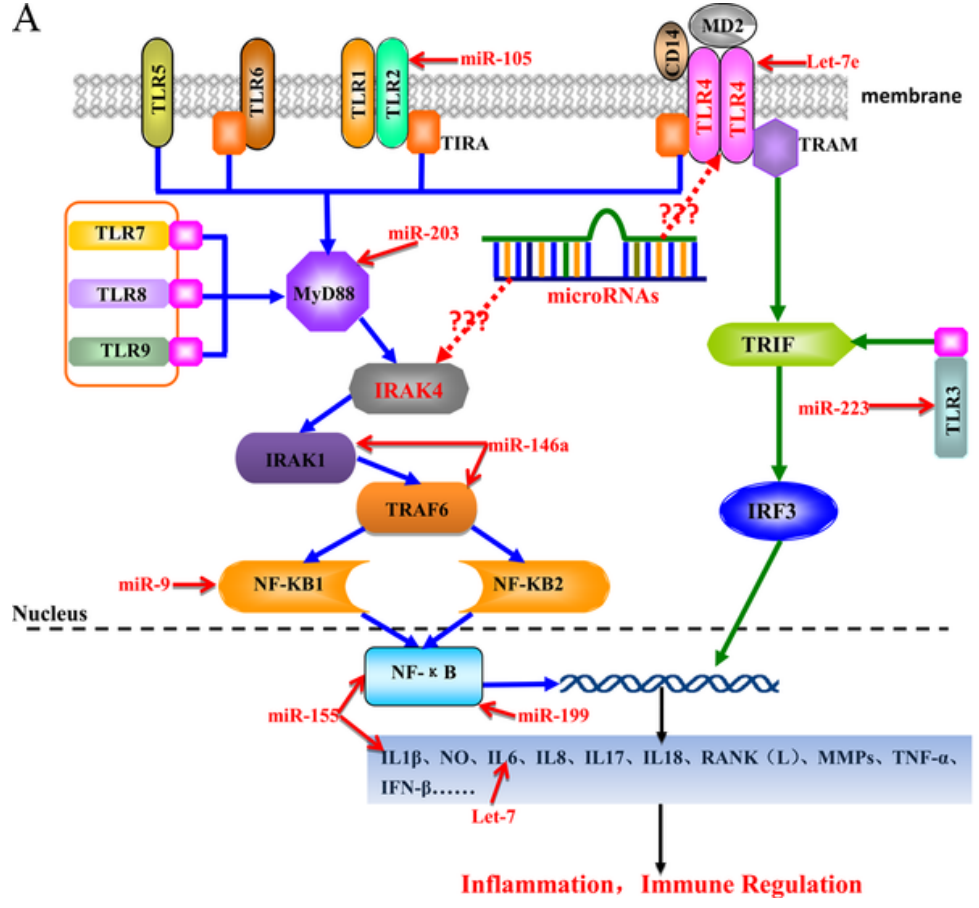

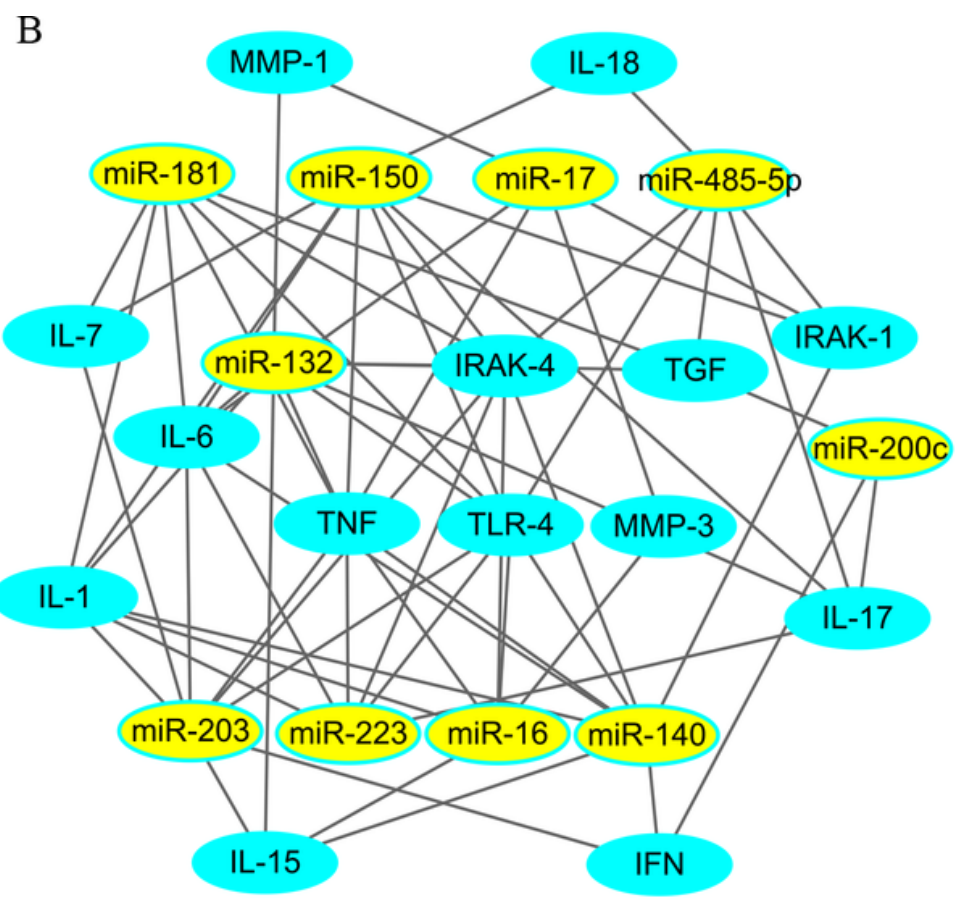

Figure 1

miRNAs are involved in inflammation. (A)miRNAs participates in TLRs/NF-kB mediated immune regulation. (B)miRNAs predicted to be associated with inflammatory factors.
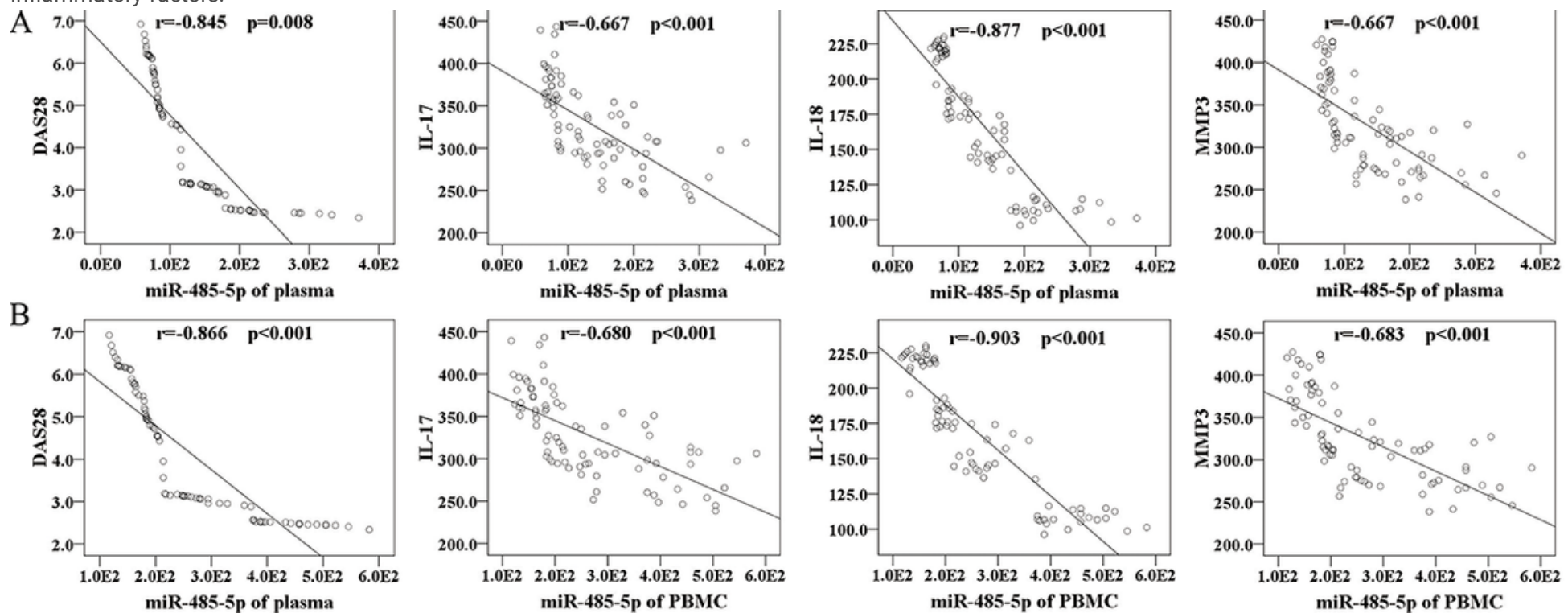

Figure 2

Correlation between clinical variables of RA and the expression of miR-485-5p. Negative correlation of DAS28, IL-17, IL-18, MMP-3 with the level of miR-485-5p in plasma $(\mathrm{A})$ and $\mathrm{PBMC}(\mathrm{B})$. 

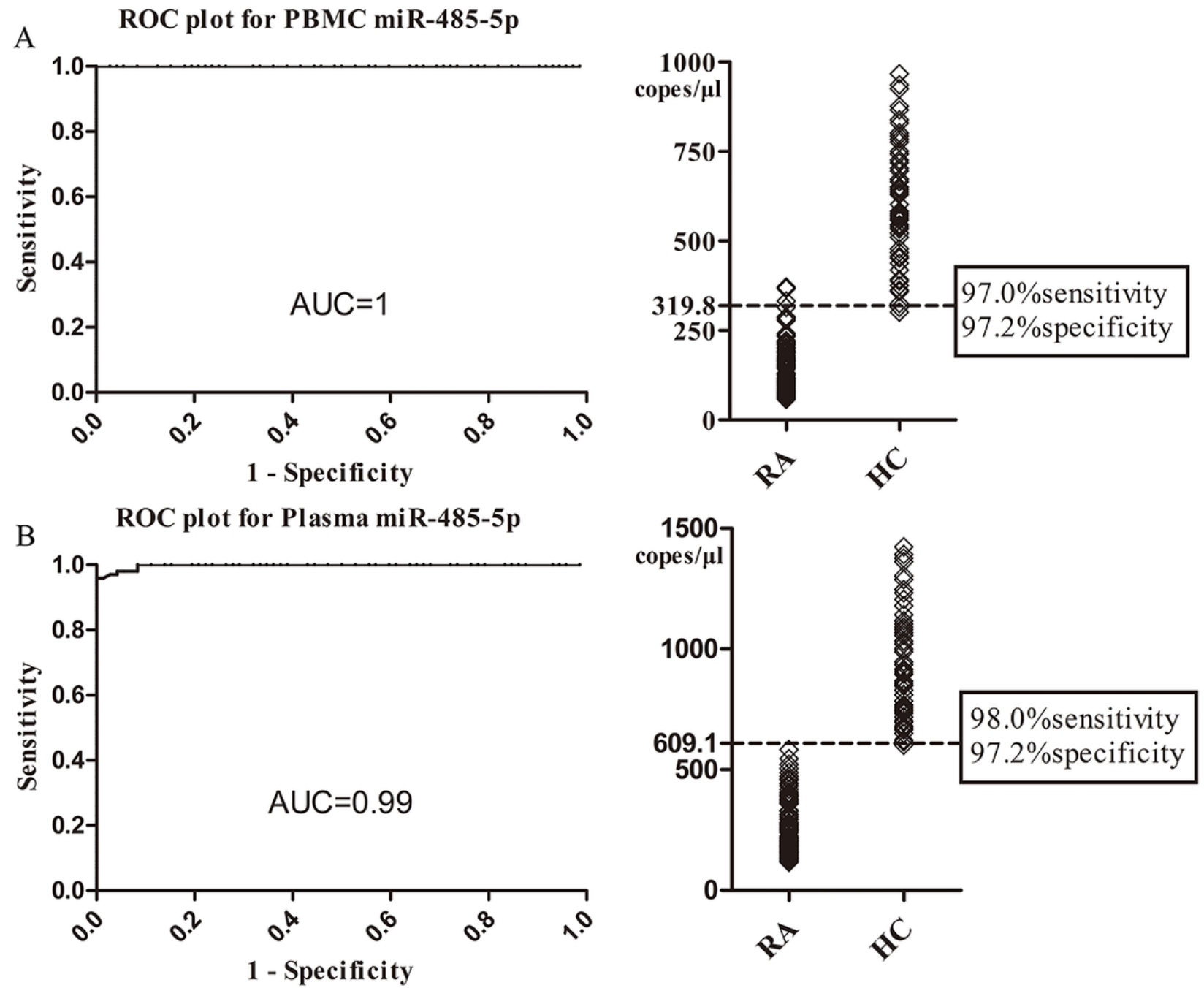

Figure 3

ROC curve analysis of plasma and PBMC miR-485-5p to differentiate patients with RA from HC. A. ROC plot of plasma miR-485-5p for the diagnosis of RA. AUC was 0.99 . A cutoff value of 319.8 copes $/ \mu$ l diagnosed RA at the sensitivity of $97.0 \%$ and the specificity of $97.2 \%$. B. ROC plot of PBMC miR-485-5p for the diagnosis of RA. AUC was 0.99. A cutoff value of $609.1 \mathrm{copes} / \mu \mathrm{l}$ diagnosed RA at the sensitivity of $98.0 \%$ and the specificity of $97.2 \%$.

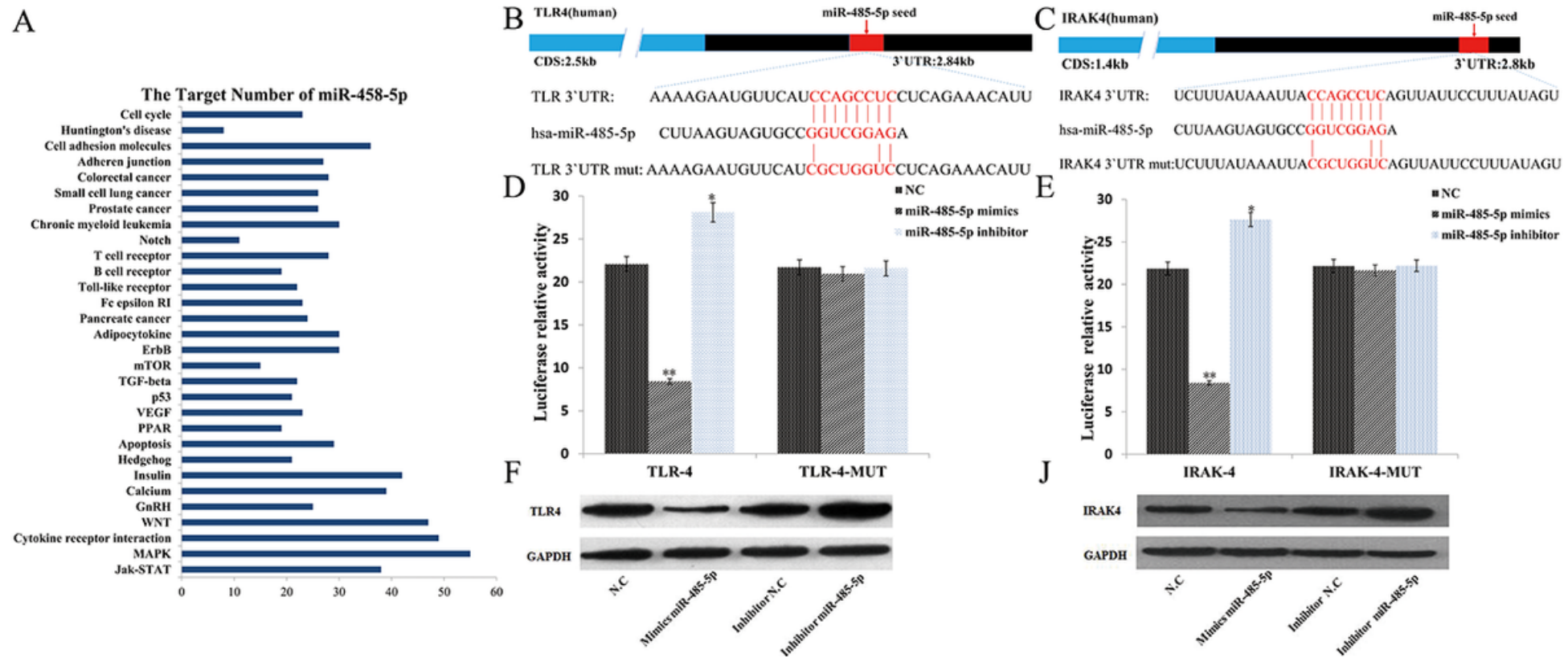


MiR-485-5p directly regulates the expression TLR-4 and IRAK-4. (A) miR-485-5p is involved in various pathways, including inflammatory reaction (B, C) Sequence alignment of miR-485-5p binding sites in the $3 U T R o f T L R-4$ and IRAK - 4. Mutantswere $\int$ roduced $\in$ theconservedb $\in d \in$ gsitesofthe 3 UTR of TLR-4 and IRAK-4. (D, E) Dual Luciferase assay for the interaction between miR-485-5p and 3'UTR of TLR-4 or IRAK-4. (F, G) The impact of miR-485-5p on the expression of TLR-4 and IRAK-4 in macrophages were determined by Western-blot. Values are MEAN \pm SD for at least three independent experiments performed in triplicate. ${ }^{*} p<0.05,{ }^{* *} p<0.01, * \star * p<0.001$ vs. control.

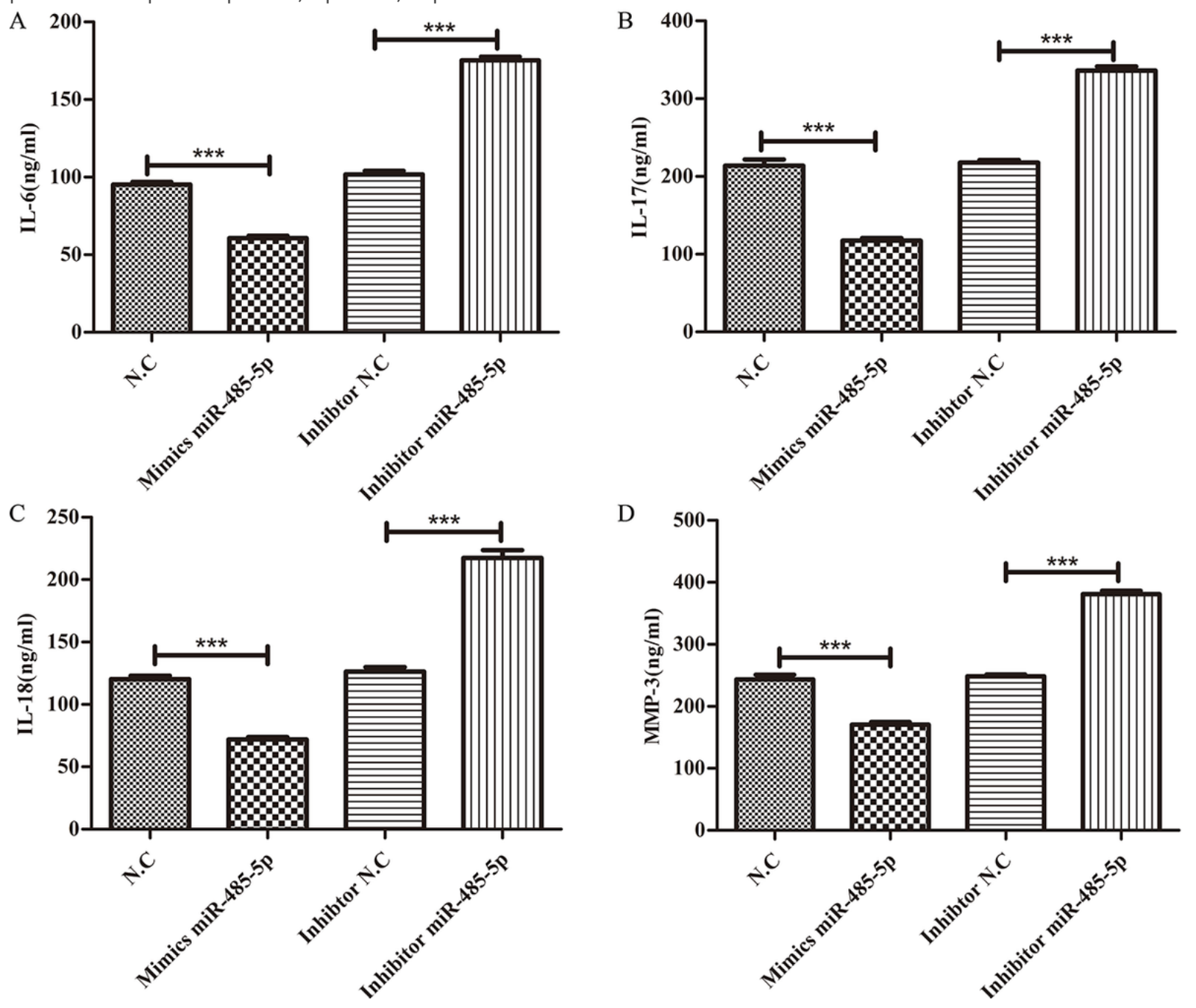

Figure 5

MiR-485-5p represses production of IL-6, IL-17, IL-18 and MMP-3. The expression of IL-6(A), IL-17(B), IL-18(C) and MMP-3(D) in macrophages trans-fected with miR-485-5p mimic or inhibitor were determined by ELISA ( $\left.{ }^{*} P<0.05, * * P<0.01\right)$. Values are MEAN \pm SD for at least three independent experiments per-formed in triplicate. ${ }^{*} p<0.05,{ }^{\star \star} p<0.01,{ }^{\star \star \star} p<0.001$ vs. control. 
A

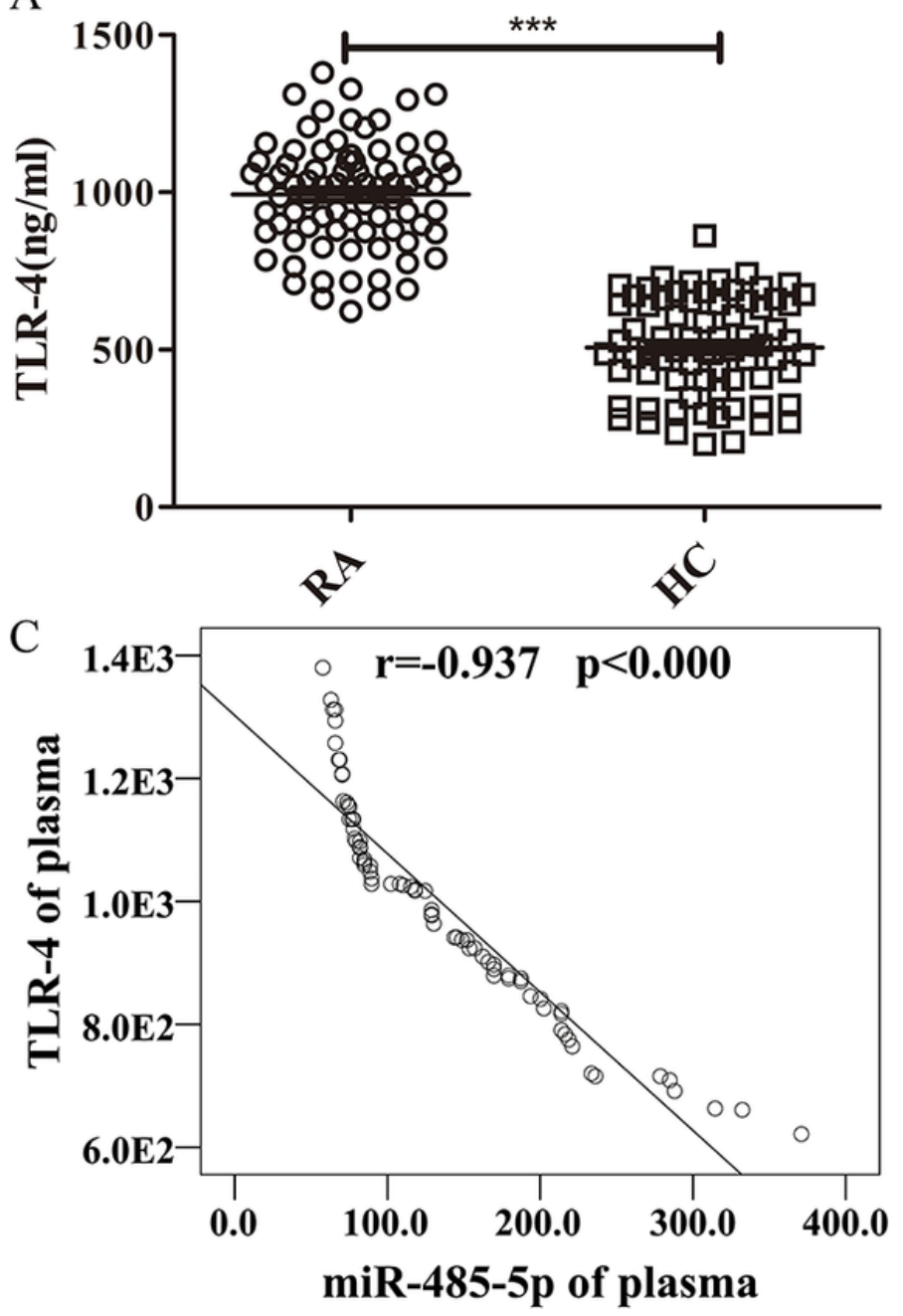

B
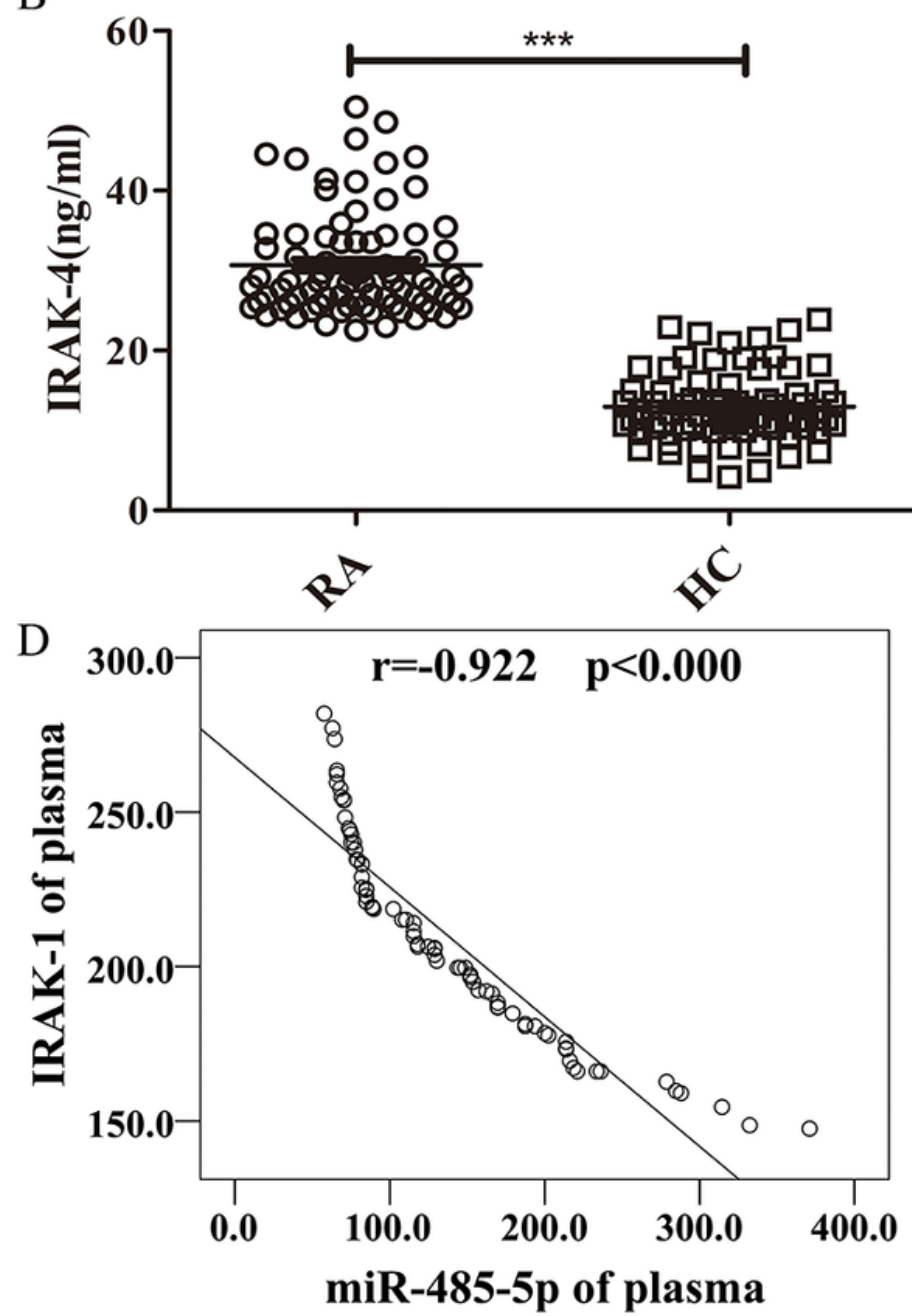

Figure 6

Correlation of the concentrations of TLR4 and IRAK4 with miR-485-5p in the plasma of RA. Plasma concentrations of TLR-4 (A) and IRAK-4 (B) in RA and HC. Correlation of plasma miR-485-5p with of TLR4 (C) and IRAK-4 (D) in RA. ***p $<0.001$ vs. con-trol. 